\title{
Role of Functional MRI in Presurgical Evaluation of Memory Function in Temporal Lobe Epilepsy
}

\author{
Chusak Limotai and Seyed M. Mirsattari \\ Departments of Clinical Neurological Sciences, Medical Biophysics, Medical Imaging and Psychology, \\ The University of Western Ontario, London, ON, Canada N6A 5A5 \\ Correspondence should be addressed to Seyed M. Mirsattari, smirsat2@uwo.ca
}

Received 16 May 2011; Revised 7 March 2012; Accepted 11 March 2012

Academic Editor: Warren T. Blume

Copyright ( 92012 C. Limotai and S. M. Mirsattari. This is an open access article distributed under the Creative Commons Attribution License, which permits unrestricted use, distribution, and reproduction in any medium, provided the original work is properly cited.

\begin{abstract}
Many diagnostic tools have been employed to predict the likelihood of a postoperative memory decline after a standard temporal lobectomy, including the intracarotid amobarbital testing (IAT) or Wada, regarded as the gold standard test for over the past half a century. Functional MRI (fMRI) is also a promising tool in that regard. Its routine use to predict the postoperative memory decline has been limited because of the varied study paradigms, discrepancies in analysis, and interpretation of the results. Based on the existing literatures, fMRI cannot replace IAT for the routine presurgical evaluation of the patients with temporal lobe epilepsy (TLE) yet. Large multicentre studies with a panel of memory test are required to determine the full potential of fMRI and use it reliably to replace IAT in the routine clinical practice. In this paper, we review various aspects of memory fMRI, including the experimental designs, data analysis, and findings.
\end{abstract}

\section{Introduction}

Epilepsy is a common chronic neurological disorder that is characterized by recurrent spontaneous seizures. Its prevalence varies between $0.5 \%$ and $1 \%$ in the general population. Epileptic seizures may be generalized or partial in onset. Temporal lobe epilepsy (TLE) is the most common partial epileptic syndrome in adult patients. Etiology of TLE is diverse, but mesial temporal sclerosis (MTS), focal cortical dysplasia, and low-grade neoplasm such as ganglioglioma or dysembryoplastic neuroepithelial tumor (DNET) account for most cases of TLE. These lesions involve amygdala, hippocampus, and parahippocampus to a variable extent (Figure 1).

The amygdala is implicated in emotional memory of fearful events, mood, and the conscious emotional response to an event. Amygdala receives its afferent inputs from the visual, auditory, and somatosensory association cortices and sends efferents to the hypothalamus and brainstem autonomic centers, including the vagal nuclei and the sympathetic neurons. The amygdala is also interconnected with the frontal cortex, the mesiodorsal thalamus, and the mesial striatum
(Figure 2). Electrical stimulation of the amygdala causes intense hallucinations that are often accompanied by fear. The hippocampus is involved in formation and retrieval of declarative memory. The hippocampus includes dentate gyrus, cornu ammonis (CA) 1-3, and subiculum (Figures 13 ). The dentate gyrus constitutes a dense dark layer of cells at the tip of the hippocampus. The subiculum is located at the base of the hippocampus in the coronal plane and is continuous with entorhinal cortex. The latter is part of the parahippocampal gyrus. Information enters the hippocampus through the perforant pathway. The entorhinal axons synapse on cells in the dentate gyrus. The axons of dentate neurons, that is, mossy fibers, end in CA3. Axons of the CA3 neurons, that is, Schaeffer collaterals, synapse on neurons in CA1, which sends its efferents to the subiculum (Figure 3). The subiculum is responsible for the output of the hippocampus. It sends its efferent axons directly to the hypothalamus and mammillary bodies via the fornix, or it passes the information back to the entorhinal cortex to relay it back to the sensory cortices. The hippocampus, CA1 in particular, is commonly involved in MTS. 


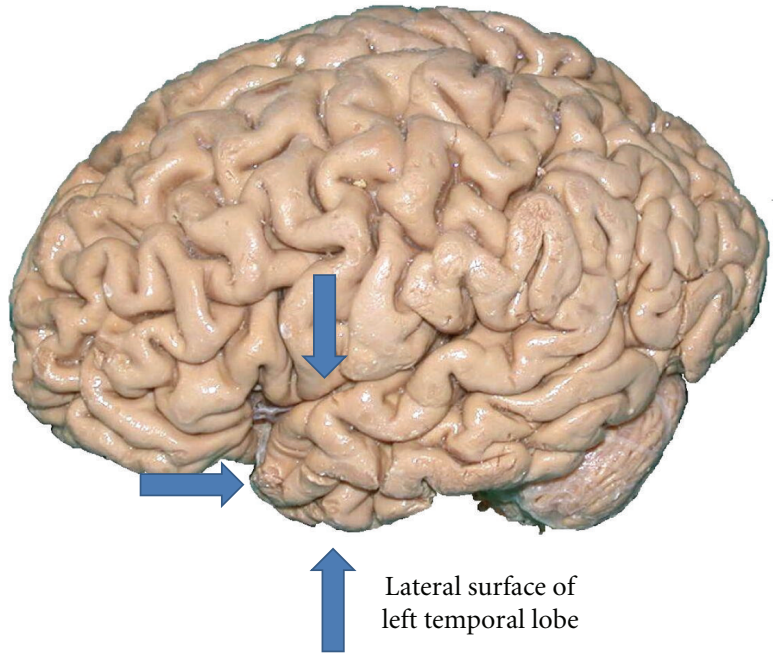

(a)

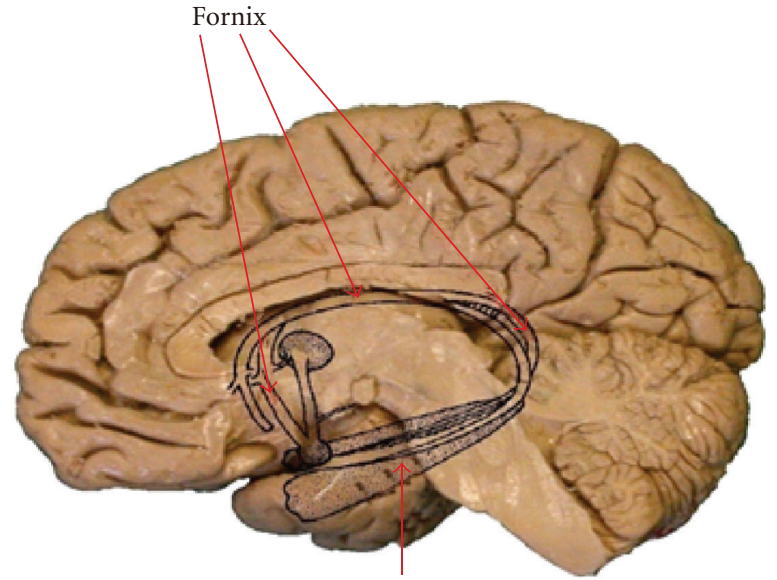

Hippocampal formation in medial temporal lobe

(b)

Figure 1: Lateral (a) and mesial (b) surfaces of temporal lobe. (Courtesy of Dr. John Kiernan, Department of Anatomy and Cell Biology, The University of Western Ontario.)

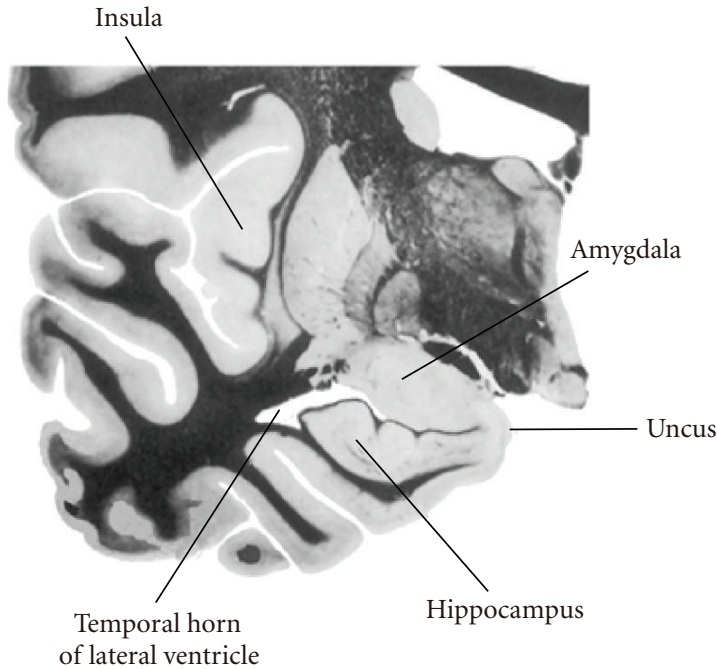

(a)
Some connections of the amygdala

Corticomedial nuclei-receive fibres from olfactory tract

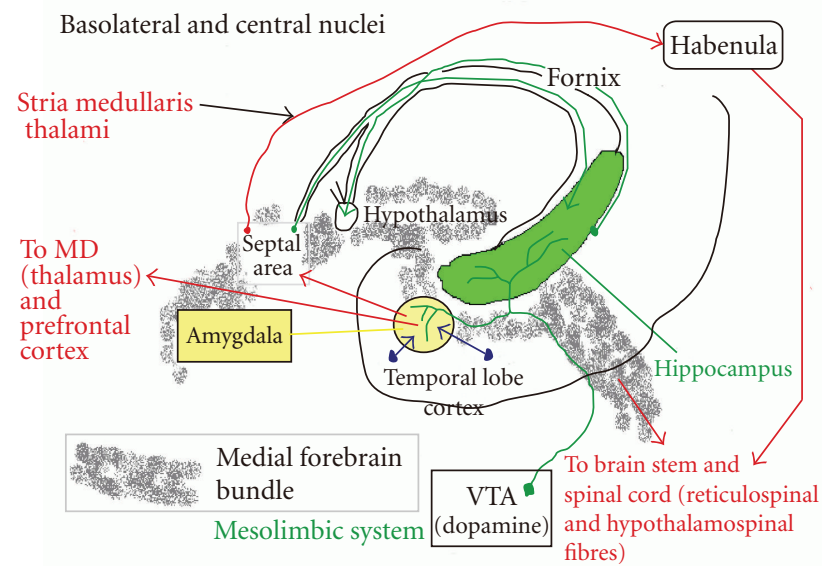

(b)

FIGURE 2: Gross coronal section of mesial temporal structures (a) and connections between amygdala and other brain regions (b). (Courtesy of Dr. John Kiernan, Department of Anatomy and Cell Biology, The University of Western Ontario.)

Different types of memory involve different neural circuitry (Figure 4). The working memory is short-term memory, which requires prefrontal cortices. The declarative memory is long-term memory, which involves personal experiences and conscious memory and requires the hippocampus for encoding. The procedural memory includes automatic actions, habits, or skills that are learned by repetition and requires the striatum and the cerebellum.

Given the overlap in declarative memory circuitry and pathology of TLE, memory impairment is common in the TLE patients. Verbal memory decline can be observed in $30-60 \%$ of the patients who undergo left anterior temporal lobectomy (ATL) [1-4]. Memory dysfunctions in TLE patients before or after temporal lobectomy (TLY) can be used to understand human memory [5-8].

Epilepsy surgery is aimed at improving seizure outcome in patients that are resistant to medical therapy. The greater the resection area, the greater is the likelihood of achieving the surgical goals. However, the extent of resection is limited by the potential cognitive deficits. Intracarotid amobarbital (IAT), also known as Wada test, is used to minimize postoperative memory and language deficits in the TLE patients. Wada test is limited to the lateralization of function than its localization, and it carries certain inherent risks for being an invasive test. Several noninvasive techniques have the potential to replace Wada as a presurgical tool to evaluate 


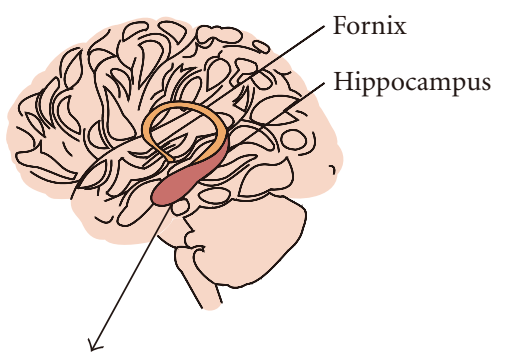

and other cognitive demands introduced by the specific task paradigms. It also depends on other factors including the field of strength of the magnet, fMRI pulse sequence, and the level of cooperation and education of the participants. This paper will begin with a review of the memory systems and their anatomical correlates. This will be followed by a discussion on memory functions subserved by medial temporal structures and related functional imaging studies, the study fMRI paradigms, the role of memory fMRI in epilepsy, particularly its role in predicting a postoperative memory

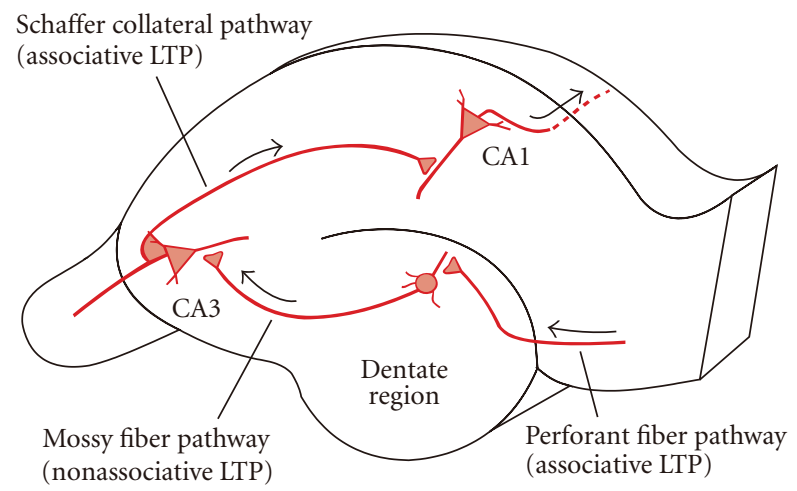
decline following TLY, and the prospect of replacing the IAT with memory fMRI.

\section{Classification of Memory Systems and Its Implication in fMRI Studies}

The memory is either explicit or implicit. Explicit memory refers to a memory in which instructions are given to remember the material during initial exposure. Implicit memory, on the other hand, refers to unintentional remembering of materials that were previously seen or learned. Conscious recollection of those learned materials is referred to as "declarative memory," whereas recollection of the skill-based information where what has been learned is embedded in acquired procedures is referred to as nondeclarative (procedural) memory [18]. Procedural memory is the memory of how to do things. When needed, procedural memories are automatically retrieved and utilized for the execution of the step-by-step procedures involved in both cognitive and motor skills.

Memory can also be defined as episodic, semantic, working, or procedural memory. Episodic memory refers to a system that records, retains, and retrieves autobiographical knowledge about experiences that occurred at a specific place and time. Semantic memory stores general conceptual and factual knowledge that is not related to any specific temporal or spatial context. Working memory is a system for temporarily storing and managing the information required to carry out complex cognitive tasks such as learning, reasoning, and comprehension. Episodic memory, semantic memory, and working memory are explicit and declarative. Procedural memory is nondeclarative and can be explicit with conscious learning and implicit by effortless learning.

Most of the fMRI studies have used explicit memoryencoding paradigms, which depend more strongly on the cognitive ability and compliance of the subjects. Few studies have used implicit memory paradigm [19] during which the subjects were not asked to remember the items and no recognition test was administered after the scanning.

\section{Memory Circuit and Neural Correlates}

The role of mesial temporal structures in memory function was demonstrated by the case of H.M. who was rendered seizure free by bilateral temporal lobectomies but was unable to convert new memories into permanent memories while his working memories, long-term memories, and procedural memories were intact [20]. Episodic memory involves 
encoding, registration, consolidation, retrieval, and reconstruction. Encoding is the process of transforming information into a format that is eventually used in long-term storage. Retrieval is the process of taking information out of the long-term storage. Mesial temporal structures, hippocampus in particular, are responsible for both encoding and retrieval processes. This explains why H.M. had marked impairment of converting short-term memory into a long-term memory. Working memory requires prefrontal cortex, whereas longterm memory is stored in the association cortices of the relevant sensory modalities (Figure 4). H.M.'s preserved implicit and procedural memory were due to his intact striatum and cerebellum [20]. Encoding and retrieval have been studied by the fMRI paradigms.

\section{Medial Temporal Lobe (MTL) Memory Functions and Related Functional Imaging Studies}

Recognition of previously experienced stimuli has two distinct underlying memory processes, which are recollection and familiarity. MTL is involved in both recollection and familiarity. The mesial temporal lobe (MTL) structures can be subdivided into perirhinal cortex, the parahippocampal cortex (called postrhinal cortex in rodents), the entorhinal cortex, and the hippocampus (Figure 5).

The hippocampus is critical for recollection but not familiarity. The parahippocampal cortex also contributes to recollection, possibly via the representation and retrieval of contextual (especially spatial) information, whereas perirhinal cortex contributes to and is necessary for familiarity [22].

The perirhinal cortex and the lateral entorhinal area encode distinct items such as people, objects, or events (i.e., "What" information). Through reciprocal connections with neocortical association areas, this can facilitate subsequent judgments of familiarity. The parahippocampal cortex and the medial entorhinal area encode contextual representations (i.e., "Where" information), while the hippocampus associates items with their context resulting in recollection upon a memory cue.

Functional imaging studies regarding subregion neuronal substrates for memory processes have shown variable results; partly because the currently used task paradigms are unable to selectively activate the region of interests. For instance, a "recollection" task paradigm may also result in some familiarity-related activation. Moreover, structures within the MTL are in close proximity of each other and most of them are highly interconnected. Thus, neural processing that originates in one region can be expected to closely activate connected neighboring regions, resulting in more extensive MRI signal changes. As a result, many authors have concluded that activity in different MTL subregions tends to be correlated with recollection and/or familiarity [22].

Dissociation in the areas of activation is observed during recollection and familiarity. Activation of the hippocampus and the parahippocampal cortex is noted during both encoding and retrieval processes. In contrast, during familiarity

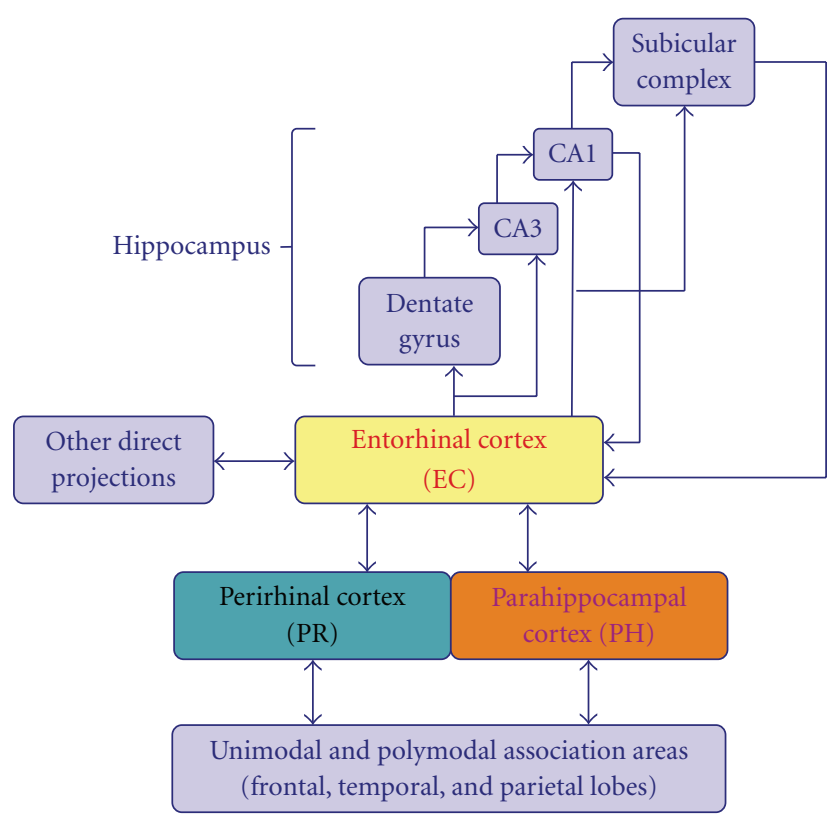

FIGURE 5: Diagram represents the MTL memory system connection for declarative memory; reciprocal connection among medial temporal subregions is demonstrated [21].

tasks, different pattern of activation is seen for encoding and retrieval processes with increased activation during encoding but reduced activation during retrieval because of increased familiarity.

There have been growing studies of functional neuroimaging including fMRI and diffusion tensor imaging (DTI) in TLE. Aside from functional mapping of the language and memory functions, many studies have demonstrated evidences of presumed epileptic networks and functional connectivity disturbance in patients with TLE. These observations provide additional support for performing functional neuroimaging studies in addition to the conventional anatomical studies, particularly in nonlesional cases. Bernhardt et al. proposed that the thalamus is an important hub in the epileptic network in TLE. They studied 36 patients with medically intractable TLE and compared them to 19 age- and sex-matched healthy controls [23]. For functional connectivity disturbance, a recent fMRI study by Morgan et al. demonstrated an increase in interhemispheric hippocampal connectivity as the epilepsy progresses longer than 10 years, even though it was initially disrupted. This is likely due to the fact that over time contralateral hippocampus exerts more influence over the ipsilateral hippocampus [24].

A recent DTI study by Keller et al. demonstrated widespread and bilaterally distributed water diffusion abnormalities, which are beyond the resolution of the conventional anatomical MRI, in patients with unilateral TLE of unknown cause. Another study revealed that DTI was able to demonstrate hippocampal abnormalities in normal-conventional MRI of the patients with TLE, which was different from those found in patients with hippocampal sclerosis (HS) [25]. 


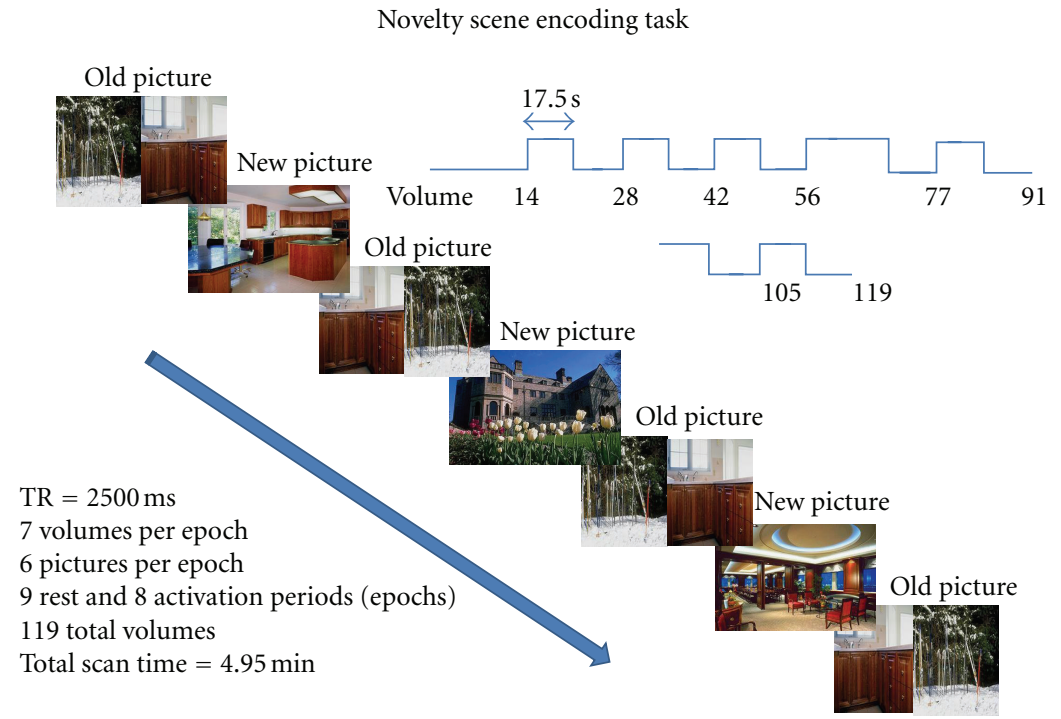

Figure 6: Novelty scene encoding task. At the time of encoding, participants are presented novel scenes that are either indoor or outdoor. During the baseline phase, repeated scenes are presented. Participants are instructed to classify the scenes as "indoor" or "outdoor."

Combining functional neuroimaging with high-field conventional anatomical MRI should increases our understanding of the epileptic and functional networks and may facilitate localization of potential epileptogenic focus.

\section{Memory Paradigms for fMRI}

The main objective of the memory fMRI in TLE is presurgical evaluation. This may help to predict the risk of memory deficits after TLY and to plan strategies that spare functional tissue. Memory fMRI may also have a predictive value for lateralization of the seizure foci in TLE. PET studies by Lepage et al. demonstrated different areas of activation within MTL, encoding in the anterior MTL, and retrieval in the posterior MTL [26]. These findings were contradictory to the fMRI findings, posterior MTL was associated with encoding [2729]. There are few fMRI studies on retrieval that show activation in the anterior MTL, particularly the subiculum [30]. The same study showed increased activation for novelty encoding in the posterior MTL, particularly the parahippocampus [30].

Most of the preoperative memory fMRI studies have employed an encoding task for the episodic memory. These studies have dealt with different aspects of episodic memory such as novelty, verbal or nonverbal tasks. Event-related or block-designed paradigms have been used in different studies.

\section{Material-Specific Memory fMRI Paradigms (Verbal versus Nonverbal)}

The lateralization of encoding process is invariably dependent on the material types. Golby et al. found that verbal encoding tasks (i.e., sentence completion) activated the inferior prefrontal cortex and the MTL more on the left side, pattern encoding (i.e., colour images of abstract patterns) more on the right side, whereas scene encoding (i.e., indoor and outdoor scenes) and faces symmetrically on both sides [31]. Powell et al. also noted that activation was left-lateralized for word encoding, bilateral for picture encoding, and right-lateralized for face encoding [32].

FMRI paradigms that produce bilateral MTL activation of MTL structures in the healthy control subjects are ideal for the presurgical evaluation of memory function in the TLE patients. Novelty scene encoding paradigm is suitable for this purpose that has shown an asymmetry of activation between the affected and unaffected MTL structures in patients with TLE [33-36] (Figures 6, 7, 8, and 9). One fMRI study using scene encoding task revealed that extent of activation within the ipsilateral MTL structures detected by fMRI during complex visual scene encoding was predictive of memory outcome [34]. This needs to be confirmed by larger studies before it can be used clinically to predict memory outcome after TLY. Greater hippocampal activation contralateral to the epileptic focus may indicate low risk of developing global amnesia. However, it is less reliable in predicting the postoperative memory deficits because the activation in that hemisphere may partly reflect brain reorganization with shift of activation from the epileptic hemisphere. The shifted activation may not necessarily represent functionally meaningful memory. In other words, a large area of activation on the contralateral side may serve as a compensatory mechanism and the patients may still have postoperative memory decline. Richardson et al. noted that activation of the right hippocampus during a verbal memory task in the left TLE patients was "dysfunctional" and it did not predict memory function postoperatively [35]. This was recently confirmed in a study by Binder et al. [36] in which they compared the predictive value of language lateralization and scene encoding 


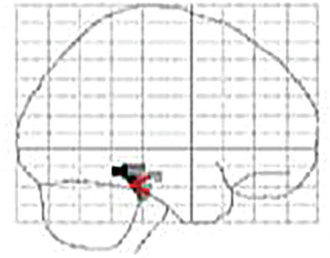

(a)

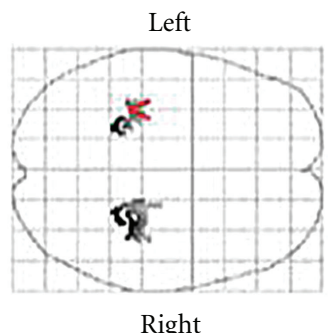

(c)

FIGURE 7: Relatively symmetrical mesial temporal structures are activated in the healthy normal control subjects during a novelty scene encoding task.

task paradigms. They found that hippocampal activation asymmetry during scene encoding was strongly correlated to the side of seizure focus and memory asymmetry scores in Wada test but it was unrelated to verbal memory outcome. Among their 30 left TLE patients, 13 had markedly discordant language and hippocampal laterality indexes (LIs). In at least five of the discordant cases, there were large memory declines despite preoperative hippocampal activation that strongly lateralized to the right. However, there was a tendency that the more area of activation on the same side as seizure focus, the more likely do the patients have postoperative memory decline. This was first observed by Rabin et al. who showed that absolute activation in the MTL structures ipsilateral to the epileptic focus had a significant negative correlation with a change in discrimination scores postoperatively. Lower activation in the epileptic temporal lobe was associated with a smaller decline or improvement in memory performance postoperatively, supporting the functional adequacy model [34].

To define functional area of verbal memory in hoping to predict the postoperative memory decline, we need a paradigm that is capable of specifically identifying this area (material-specific paradigm). Verbal memory encoding paradigm is a good option. Richardson et al. showed that right-handed patients with left hippocampal sclerosis preferentially activate the right hippocampal formation in a verbal memory encoding task. The distribution of activity between left and right hippocampi during this verbal encoding task reflects the severity of left hippocampal sclerosis [37]. They subsequently showed that relatively greater verbal memory encoding activation in left hippocampus compared to the right hippocampus predicted the extent of verbal memory decline in right-handed patients with left hippocampal sclerosis undergoing left anterior temporal lobe resection [38].

A recent study by Bonelli et al. showed that among 72 patients with unilateral mTLE (41 left) and 20 healthy controls who underwent memory encoding for pictures, words, and faces. They reported greater left than right anterior hippocampal activation that in left TLE patients on word encoding correlated with greater verbal memory decline after left anterior TLY, while greater right than left anterior hippocampal activation on face encoding predicted greater visual memory decline after right anterior TLY. This supports the benefit of using purely verbal memory paradigm as words and purely nonverbal memory paradigm as face (or pattern) in predicting verbal and nonverbal memory decline in left and right mTLE, respectively [39].

Binder et al. carried out a preoperative language mapping to predict postoperative verbal memory decline. They demonstrated that language lateralization measured was the second most powerful predictor for verbal memory decline (added 10\% additional predictive power), for which preoperative memory performance was accounted for the most powerful predictor. Patients with higher preoperative test scores showed larger declines. Whereas neither Wada memory asymmetry, nor did Wada language asymmetry have additional predictive values beyond other noninvasive measures [40].

\section{Block Design and Event-Related Paradigms}

Even though several studies have shown predictive value of performing fMRI with encoding memory paradigm to predict postoperative memory decline, one fact should be kept in mind is that these encoding-related activation was localized to the posterior hippocampus and parahippocampal regions, not anterior MTL structures, which is resected during a standard TLY. Most studies which have used block designs have generally revealed more posterior mesial temporal activations in individual subjects $[33,41]$. Therefore, ideally, a paradigm that is able to activate the anterior temporal structures should be an optimal paradigm for this purpose.

The more anterior mesial temporal activations, particularly the hippocampal activations, have so far been reported at group levels, especially when event-related designs were used. Constable et al. demonstrated that the block design paradigm provides strong posterior activation, likely related more to visual scene processing, whereas the event-related design provides more anterior hippocampal activation with the encoding of novel scenes [42].

Powell et al. demonstrated that only event-related studies allow the identification of brain regions showing greater activation during the encoding of items that are subsequently remembered compared with items that are forgotten, which are then taken as candidate neural correlates of memory function. They argue that event-related paradigm is more 


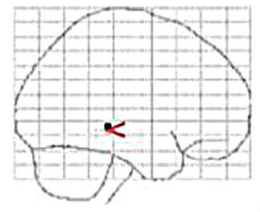

(a)
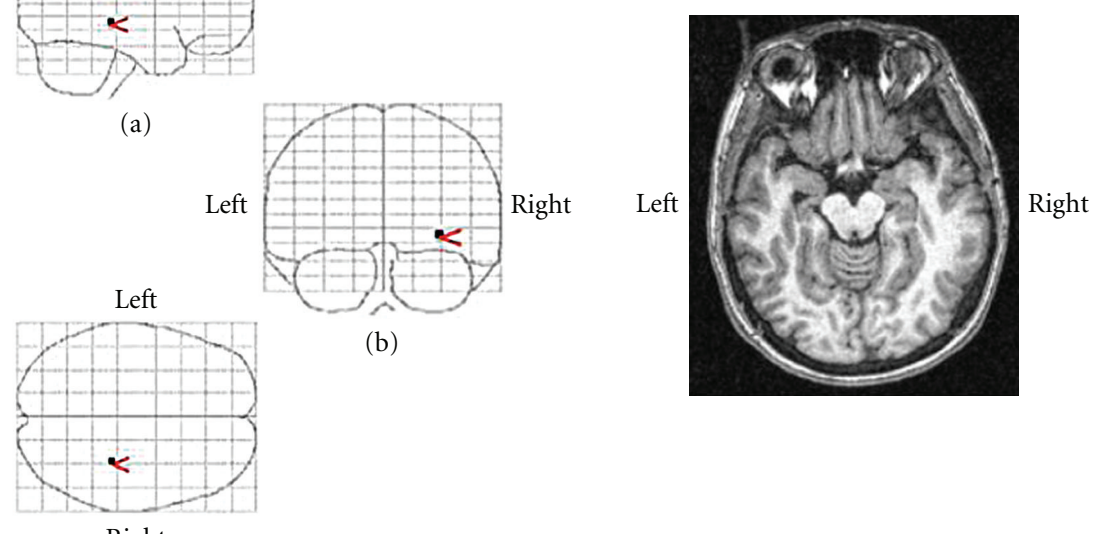

Right

(c)

(A)

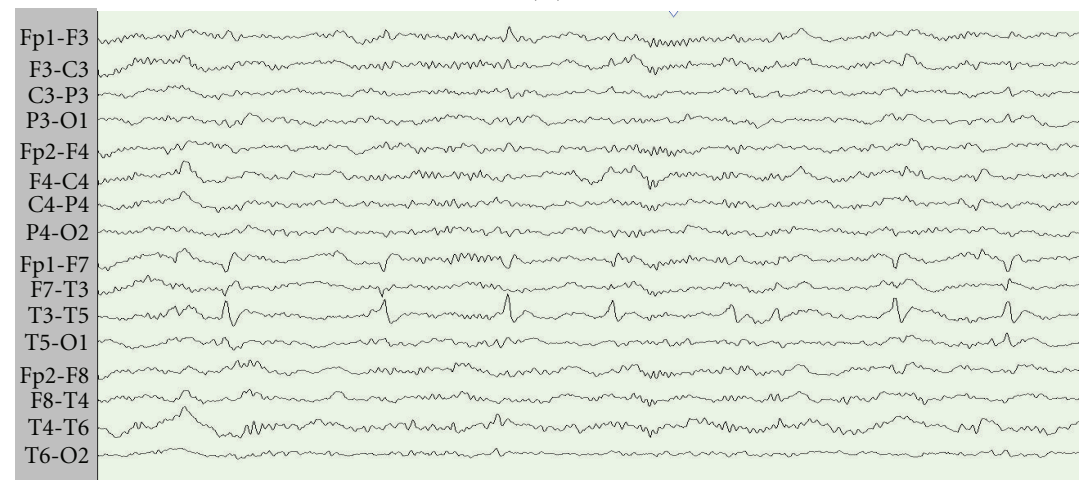

(a)

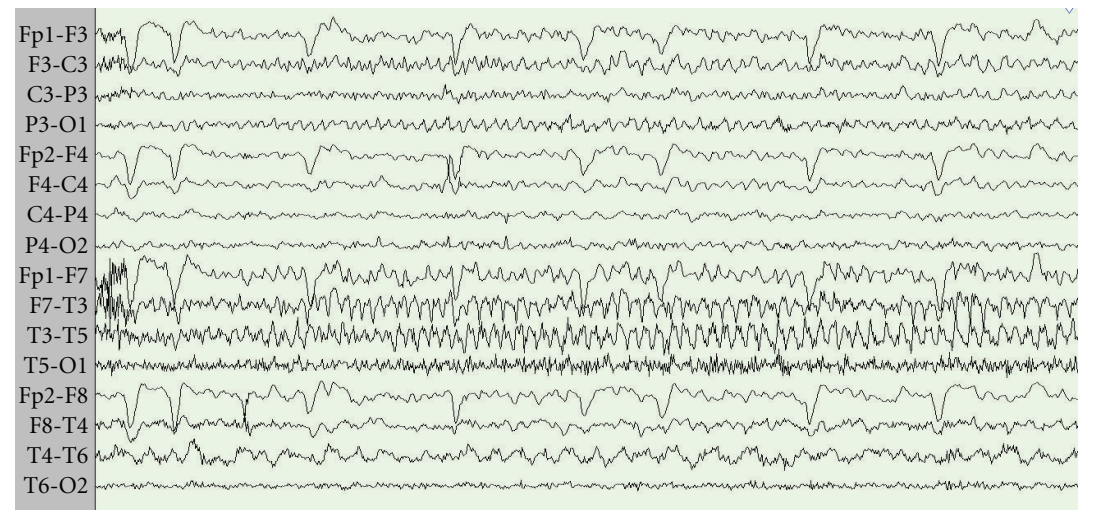

(b)

(B)

FIgURE 8: (A) Unilateral activation of the right mesial temporal structures in a patient with left mesial temporal sclerosis, during the novelty scene encoding task. (B) Left anterior to mid-temporal interictal epileptiform discharges (a) in the same patient as (A), and ictal EEG revealed left temporal originating seizure (b).

reliable than block designs, revealing anterior hippocampal activation. However, there is a relatively less activation with event-related paradigms than block design paradigms [32]. Autobiography memory retrieval task is one of the promising event-related paradigms that has been shown to demonstrate discernible activation in the memory network [43]. This may be employed in the future study to predict the postoperative memory decline. 


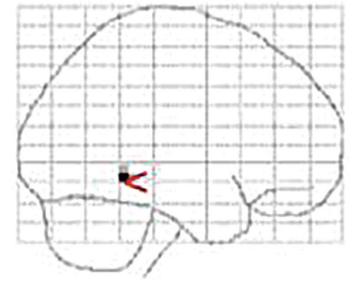

(a)
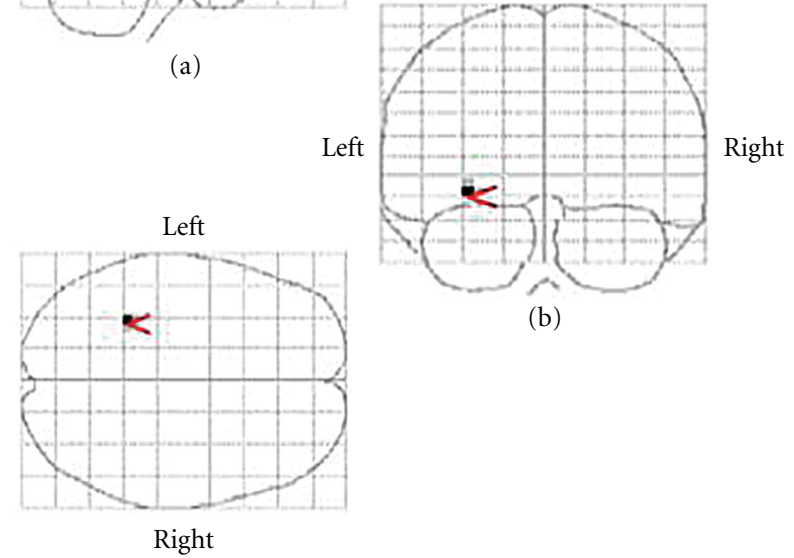

(b)

(c)

FIgURE 9: Unilateral left mesial temporal structures activation in the patient with right mesial temporal sclerosis, during novelty scene encoding task.

\section{Novelty and Repeated Tasks (Familiarized) Paradigms}

Some earlier studies failed to show activation within the MTL structures during a novelty encoding task [44, 45]. Henke et al. found hippocampal and parahippocampal activation only during associative learning and it was not seen during other tasks including novelty encoding.

Subsequently, in a study by Gabrieli et al., five of six subjects showed greater activation in the posterior MTL location for novelty encoding scenes. They concluded that parahippocampal activation could reflect memory processes that distinguish between familiar and unfamiliar stimuli [30]. Many subsequent studies have supported the possibility that the activation associated with novelty occurs more often in the posterior parahippocampus and adjacent fusiform gyrus than in the hippocampus proper [27, 46, 47].

However, a number of other imaging studies have shown stronger hippocampal activation for meaningful relative to meaningless stimuli and associative/semantic relative to nonsemantic tasks. Hunkin et al. demonstrated that mesial temporal novelty-related activations occurred for novel verbal associations (subjects were required to generate a vivid, imaginable sentence linking the three words given together), but not for novel verbal items. Binder et al. studied hippocampal activation patterns during encoding protocols emphasizing either novelty or relational processing. In the novelty contrast, they compared activation between encoding of novel scenes and a repeated pairs of scenes. In the relational processing contrast, to exclusively elucidate the effect of relational process, they compared between encoding novel scenes and structural encoding of scrambled scenes. They found that

the relational processing protocol resulted in a larger volume of hippocampal activation when compared with the novelty contrast protocol.

Novelty scene encoding paradigm has been used in most fMRI studies. Some of these studies employed scrambled images as their control, while others have used repeated scenes. We have adopted the latter protocol using repeated scenes as controls at our centre (Figure 6). Lately, there was a study directly comparing the hippocampal activation patterns of two paradigms, the authors found that the relational processing contrast encoding of novel scenes compared with structural encoding of scrambled images control design resulted in a larger volume of hippocampal activation, as compared to the novelty contrast encoding of novel scenes compared with encoding of a repeated pair of scenes [48]. However, this has to be proved by further studies.

\section{Predictive Value of fMRI for Memory Decline after Temporal Lobectomy}

There has been some progress in using preoperative fMRI to predict postoperative verbal memory decline. However, this is of limited clinical utility in patients with nondominant TLE where postoperative neuropsychological testing shows no deficits or improvement in verbal memory because patients are on less medication. There is still controversy on the presence of fMRI paradigm(s) that can be equally used in patients with dominant and nondominant TLE. It remains unclear on how to interpret the asymmetry in fMRI activation patterns. More contralateral activation than ipsilateral activation may support the functional reserve model, while unilateral activation on the ipsilateral side may support the functional adequacy model. Hippocampal reserve refers to the capacity of the contralateral hippocampus to support memory after surgery, while the functional adequacy refers to a possible significant decline in memory after ATL in patients with a higher baseline memory performance than those with lower baseline memory performance.

Most of the memory fMRI studies have been supportive of functional adequacy model, implying that individuals with greater ipsilateral activation compared with the contralateral mesial temporal activation have a greater memory decline following temporal lobectomy [39, 40, 49-51]. Similarly, Rabin et al. showed a significant inverse correlation between ipsilateral activation and memory outcome after TLY [34]. Additionally, Powell et al. demonstrated that reorganization of the intact MTL was incapable of preserving memory function [52]. Memory and language fMRI studies in prediction of postoperative verbal memory decline following a temporal lobectomy are summarized and shown in Table 1.

\section{Can fMRI Replace the Intracarotid Amobarbital Test (IAT) for Presurgical Evaluation of Memory Function?}

The intracarotid amobarbital (IAT), also known as Wada test, is an invasive procedure with a potential for complications 
TABLE 1: Memory and language fMRI studies in prediction of postoperative verbal memory decline following a temporal lobectomy.

\begin{tabular}{|c|c|c|c|c|c|}
\hline Authors & Year & Number & Memory paradigms & Summary & Remarks \\
\hline Rabin et al. [34] & 2004 & $35(20 \mathrm{Rt} / 15 \mathrm{Lt})$ & $\begin{array}{l}\text { Complex visual } \\
\text { scene encoding }\end{array}$ & $\begin{array}{l}\text { Significant inverse correlation between } \\
\text { activation ipsilateral to temporal } \\
\text { lobectomy and memory outcome. } \\
\text { No significant correlation in the } \\
\text { contralateral activation }\end{array}$ & $\begin{array}{l}\text { fMRI ARs correlated } \\
\text { significantly with } \\
\text { memory lateralization } \\
\text { by IAT }\end{array}$ \\
\hline Richardson et al. [37] & 2004 & $10($ all Lt $)$ & $\begin{array}{l}\text { Verbal memory } \\
\text { encoding }\end{array}$ & $\begin{array}{l}\text { Relatively greater verbal memory } \\
\text { encoding activity in left HC compared } \\
\text { with right } \mathrm{HC} \text {, as measured using fMRI, } \\
\text { predicts the extent of verbal memory } \\
\text { decline in the same subjects }\end{array}$ & Material specific (verbal) \\
\hline Richardson et al. [35] & 2006 & $\begin{array}{c}30 \text { (all Lt, } 12 \\
\text { underwent ATL) }\end{array}$ & $\begin{array}{l}\text { Verbal memory } \\
\text { encoding }\end{array}$ & $\begin{array}{l}\text { Functional adequacy of left HC best } \\
\text { predicts postoperative memory } \\
\text { outcome in left HS }\end{array}$ & $\begin{array}{l}\text { Material specific } \\
\text { (verbal), event related }\end{array}$ \\
\hline Binder et al. [40] & 2008 & $60($ all Lt) & Language & $\begin{array}{l}\text { Lateralization of language is correlated } \\
\text { with lateralization of verbal memory }\end{array}$ & $\begin{array}{l}\text { Wada memory testing is } \\
\text { insufficiently reliable }\end{array}$ \\
\hline Powell et al. [49] & 2008 & $15(7 \mathrm{Rt} / 8 \mathrm{Lt})$ & $\begin{array}{l}\text { Encoding of words } \\
\text { (verbal), pictures, } \\
\text { and faces }\end{array}$ & $\begin{array}{l}\text { Relatively greater ipsilateral activation } \\
\text { had greater memory decline }\end{array}$ & $\begin{array}{l}\text { Supports the functional } \\
\text { adequacy theory of } \mathrm{HC} \\
\text { function }\end{array}$ \\
\hline Frings et al. [50] & 2008 & $22(10 \mathrm{Rt} / 12 \mathrm{Lt})$ & $\begin{array}{l}\text { Encoding and } \\
\text { recognition of } \\
\text { object locations }\end{array}$ & $\begin{array}{l}\text { Positive correlation between the } \\
\text { degree of ipsilaterality lateralized HC } \\
\text { activation and postsurgical verbal } \\
\text { memory decline }\end{array}$ & $\begin{array}{l}\text { Nonverbal paradigm } \\
\text { predicts postsurgical } \\
\text { verbal memory decline }\end{array}$ \\
\hline Binder et al. [36] & 2010 & $67(37 \mathrm{Rt} / 30 \mathrm{Lt})$ & $\begin{array}{l}\text { Language and scene } \\
\text { memory encoding }\end{array}$ & $\begin{array}{l}\text { Risk of verbal memory decline is more } \\
\text { closely correlated with language } \\
\text { lateralization than with overall } \\
\text { asymmetry of episodic memory } \\
\text { processes }\end{array}$ & $\begin{array}{l}\text { Language paradigm to } \\
\text { predict verbal memory } \\
\text { decline }\end{array}$ \\
\hline Bonelli et al. [39] & 2010 & $72(31 \mathrm{Rt} / 41 \mathrm{Lt})$ & $\begin{array}{l}\text { Encoding of words } \\
\text { (verbal), pictures, } \\
\text { and faces }\end{array}$ & $\begin{array}{l}\text { Preoperative memory fMRI was } \\
\text { the strongest predictor of verbal } \\
\text { and visual memory decline }\end{array}$ & $\begin{array}{l}\text { Memory fMRI in } \\
\text { prediction of both } \\
\text { postsurgical verbal and } \\
\text { visual memory decline }\end{array}$ \\
\hline Dupont et al. [51] & 2010 & $25(14 \mathrm{Rt} / 11 \mathrm{Lt})$ & $\begin{array}{l}\text { Scene encoding } \\
\text { and recognition }\end{array}$ & $\begin{array}{l}\text { fMRI activation during a } \\
\text { delayed-recognition task is a better } \\
\text { predictor of individual postoperative } \\
\text { verbal memory outcome than is the } \\
\text { Wada test }\end{array}$ & $\begin{array}{l}\text { Only one study revealed } \\
\text { marked discordant LI } \\
\text { between memory fMRI } \\
\text { and Wada test (only } 48 \% \\
\text { of the patients showed } \\
\text { concordant result) }\end{array}$ \\
\hline
\end{tabular}

Rt: right; Lt: left; AR: asymmetry ratio; IAT: intracarotid sodium amytal test; HC: hippocampus; ATL: anterior temporal lobectomy; HS: hippocampal sclerosis; LI: laterality index.

such as stroke or death [53]. Predictive value of IAT for postoperative memory decline is not conclusive. Since its first use by Wada to lateralize language function in 1950s $[54,55]$ and Milner to predict the postoperative decline in memory after TLY [56], the predictive accuracy and added values of IAT to other noninvasive data remain controversial. It has been shown that IAT was of no added benefits to predict postoperative memory decline to a comprehensive preoperative neuropsychological evaluation $[2,4,57-59]$, age at onset of epilepsy $[60,61]$, plus high resolution anatomical MRI in left TLE $[59,62,63]$, even though is considered the gold standard test. Several advanced noninvasive technologies including fMRI have the potential to replace IAT. Additionally, it is still debated on how to interpret the IAT results. Some authors support the functional reserve model $[57,64]$, whereas many others support the functional adequacy model $[2,65,66]$.
Over the past decade, all of these have led to a question that it is time to replace Wada test with other techniques [67-70].

10.1. Concordance between $f M R I$ and IAT Results. In a study by Detre et al., there was 100\% concordance between fMRI and IAT in nine patients [33]. Deblaere et al. demonstrated that fMRI activation pattern in the MTL that lateralized to the contralateral hemisphere was stronger than the IAT result, that is, relatively higher laterality index (LI) from the fMRI result when compared with IAT. This might help better determine the side of seizure focus with certainty. However, the side of lateralization obtained from fMRI was in agreement with IAT results in only those patients with right-sided TLE [35]. Binder et al. noted that the anterior hippocampus LI and whole hippocampus LI were significantly correlated 
with Wada memory asymmetry in either left- or right-sided TLE [36]. Over the past decade, most of the studies have shown that memory asymmetry with fMRI is concordant to the memory asymmetry scores in IAT. Dupont et al. demonstrated striking discordance between fMRI LI and Wada LI. Only 48\% (12) of their 25 patients showed concordance between fMRI LI and Wada LI. They noted fMRI was better than IAT in accurately predicting postoperative memory changes [51].

\section{Summary}

Memory decline is a major concern after TLY. With optimal study paradigms and higher resolution MR scanners, fMRI has the potential to replace IAT to evaluate this risk noninvasively. Given the diverse nature of human memory and multiple roles served by the temporal lobes for this function, a panel of tests is necessary to probe each aspect of the memory, including verbal, nonverbal, encoding, and retrieval. Sentence completion task and naming paradigms are better suited to study verbal memory, while face recognition is better suited for nonverbal memory. Novelty scene paradigm and autobiographical memory result in a relatively symmetrical activation bilaterally and each tests a unique feature of temporal lobe function, that is, encoding and retrieval, respectively. Given the inherent limitations to perform fMRI in patients with epilepsy such as fatigue, possible seizures during the study, poor concentration, and so forth paradigms with higher specificity and sensitivity should be utilized. Novelty scene encoding and autobiographical memory have the potential to serve this role but further studies in larger population are required to validate this. Language paradigms have shown promising results in predicting verbal memory decline after TLY especially in dominant hemisphere for language function. Paradigms that result in greater activation of the anterior MTL are ideal because standard TLY removes the anterior portions of temporal lobes. Novelty scene encoding results in greater activation of the mid to posterior regions of the MTL. The role of language fMRI to predict postoperative decline in verbal memory is promising. The prognostic accuracy of fMRI to aid in prediction of postoperative memory changes is equal to better than that of the IAT.

\section{Acknowledgments}

The authors wish to thank Dr. John Kiernan, Frank Bihari, Chukiat Limotai, and Dr. Natlada Limotai for preparing figures and providing them helpful suggestions.

\section{References}

[1] C. Helmstaedter and C. E. Elger, "Cognitive consequences of two-thirds anterior temporal lobectomy on verbal memory in 144 patients: a three-month follow-up study," Epilepsia, vol. 37, no. 2, pp. 171-180, 1996.

[2] E. Stroup, J. Langfitt, M. Berg, M. McDermott, W. Pilcher, and P. Como, "Predicting verbal memory decline following anterior temporal lobectomy (ATL)," Neurology, vol. 60, no. 8, pp. 1266-1273, 2003.

[3] U. Gleissner, C. Helmstaedter, J. Schramm, and C. E. Elger, "Memory outcome after selective amygdalohippocampectomy in patients with temporal lobe epilepsy: one-year follow-up," Epilepsia, vol. 45, no. 8, pp. 960-962, 2004.

[4] S. Baxendale, P. Thompson, W. Harkness, and J. Duncan, "Predicting memory decline following epilepsy surgery: a multivariate approach," Epilepsia, vol. 47, no. 11, pp. 1887-1894, 2006.

[5] B. Bowles, C. Crupi, S. M. Mirsattari et al., "Impaired familiarity with preserved recollection after anterior temporal-lobe resection that spares the hippocampus," Proceedings of the National Academy of Sciences of the United States of America, vol. 104, no. 41, pp. 16382-16387, 2007.

[6] B. Bowles, E. B. O’Neil, S. M. Mirsattari, J. Poppenk, and S. Köhler, "Preserved hippocampal novelty responses following anterior temporal-lobe resection that impairs familiarity but spares recollection," Hippocampus, vol. 21, no. 8, pp. 847-854, 2011.

[7] C. B. Martin, B. Bowles, S. M. Mirsattari, and S. Köhler, "Selective familiarity deficits after left anterior temporal-lobe removal with hippocampal sparing are material specific," Neuropsychologia, vol. 49, no. 7, pp. 1870-1878, 2011.

[8] E. C. Leritz, L. J. Grande, and R. M. Bauer, "Temporal lobe epilepsy as a model to understand human memory: the distinction between explicit and implicit memory," Epilepsy and Behavior, vol. 9, no. 1, pp. 1-13, 2006.

[9] A. M. Dale and E. Halgren, "Spatiotemporal mapping of brain activity by integration of multiple imaging modalities," Current Opinion in Neurobiology, vol. 11, no. 2, pp. 202-208, 2001.

[10] R. J. Ilmoniemi, J. Ruohonen, and J. Karhu, "Transcranial magnetic stimulation - a new tool for functional imaging of the brain," Critical Reviews in Biomedical Engineering, vol. 27, no. 3-5, pp. 241-284, 1999.

[11] J. R. Binder, S. J. Swanson, T. A. Hammeke et al., "Determination of language dominance using functional MRI: a comparison with the Wada test," Neurology, vol. 46, no. 4, pp. 978-984, 1996.

[12] X. Gao, C. Jiang, C. Lu, and T. Shen, "Determination of the dominant language hemisphere by functional MRI in patients with temporal lobe epilepsy," Chinese Medical Journal, vol. 114, no. 7, pp. 711-713, 2001.

[13] P. Sabbah, F. Chassoux, C. Leveque et al., "Functional MR imaging in assessment of language dominance in epileptic patients," NeuroImage, vol. 18, no. 2, pp. 460-467, 2003.

[14] G. J. M. Rutten, N. F. Ramsey, P. C. Van Rijen, W. C. Alpherts, and C. W. M. Van Veelen, "fMRI-determined language lateralization in patients with unilateral or mixed language dominance according to the Wada test," NeuroImage, vol. 17, no. 1, pp. 447-460, 2002.

[15] T. Benke, B. Köylü, P. Visani et al., "Language lateralization in temporal lobe epilepsy: a comparison between fMRI and the Wada test," Epilepsia, vol. 47, no. 8, pp. 1308-1319, 2006.

[16] J. Arora, K. Pugh, M. Westerveld, S. Spencer, D. D. Spencer, and R. Todd Constable, "Language lateralization in epilepsy patients: FMRI validated with the Wada procedure," Epilepsia, vol. 50, no. 10, pp. 2225-2241, 2009.

[17] D. S. Sabsevitz, S. J. Swanson, T. A. Hammeke et al., "Use of preoperative functional neuroimaging to predict language deficits from epilepsy surgery," Neurology, vol. 60, no. 11, pp. 1788-1792, 2003. 
[18] A. E. Budson and B. H. Price, "Current concepts: memory dysfunction," New England Journal of Medicine, vol. 352, no. 7, pp. 692-699, 2005.

[19] B. Weber, F. Kügler, and C. E. Elger, "Comparison of implicit memory encoding paradigms for the activation of mediotemporal structures," Epilepsy and Behavior, vol. 10, no. 3, pp. 442448, 2007.

[20] E. R. Kandel, "Different memories, different brain regions," in In Search of Memory: The Emergence of a New Science of Mind, E. R. Kandel, Ed., pp. 127-133, W. W. Norton \& Company, New York, NY, USA, 2006.

[21] L. R. Squire and J. T. Wixted, "The cognitive neuroscience of human memory since H.M.," Annual Review of Neuroscience, vol. 34, pp. 259-288, 2011.

[22] H. Eichenbaum, A. P. Yonelinas, and C. Ranganath, "The medial temporal lobe and recognition memory," Annual Review of Neuroscience, vol. 30, pp. 123-152, 2007.

[23] B. C. Bernhardt, N. Bernasconi, H. Kim et al., "Mapping thalamocortical network pathology in temporal lobe epilepsy," Neurology, vol. 78, no. 2, pp. 129-136, 2012.

[24] V. L. Morgan, B. P. Rogers, H. H. Sonmezturk, J. C. Gore, and B. Abou-Khalil, "Cross hippocampal influence in mesial temporal lobe epilepsy measured with high temporal resolution functional magnetic resonance imaging," Epilepsia, vol. 52, no. 9, pp. 1741-1749, 2011.

[25] S.S. Keller, T. Ahrens, S. Mohammadi et al., "Voxel-based statistical analysis of fractional anisotropy and mean diffusivity in patients with unilateral temporal lobe epilepsy of unknown cause," Journal of Neuroimaging. In press.

[26] M. Lepage, R. Habib, and E. Tulving, "Hippocampal PET activations of memory encoding and retrieval: the HIPER model," Hippocampus, vol. 8, no. 4, pp. 313-322, 1998.

[27] C. E. Stern, S. Corkin, R. G. González et al., "The hippocampal formation participates in novel picture encoding: evidence from functional magnetic resonance imaging," Proceedings of the National Academy of Sciences of the United States of America, vol. 93, no. 16, pp. 8660-8665, 1996.

[28] G. Fernández, H. Weyerts, M. Schrader-Bölsche et al., "Successful verbal encoding into episodic memory engages the posterior hippocampus: a parametrically analyzed functional magnetic resonance imaging study," Journal of Neuroscience, vol. 18, no. 5, pp. 1841-1847, 1998.

[29] D. L. Schacter and A. D. Wagner, "Medial temporal lobe activations in fMRI and PET studies of episodic encoding and retrieval," Hippocampus, vol. 9, no. 1, pp. 7-24, 1999.

[30] J. D. E. Gabrieli, J. B. Brewer, J. E. Desmond, and G. H. Glover, "Separate neural bases of two fundamental memory processes in the human medial temporal lobe," Science, vol. 276, no. 5310, pp. 264-266, 1997.

[31] A. J. Golby, R. A. Poldrack, J. B. Brewer et al., "Material-specific lateralization in the medial temporal lobe and prefrontal cortex during memory encoding," Brain, vol. 124, no. 9, pp. 1841-1854, 2001.

[32] H. W. R. Powell, M. J. Koepp, M. R. Symms et al., "Materialspecific lateralization of memory encoding in the medial temporal lobe: blocked versus event-related design," NeuroImage, vol. 27, no. 1, pp. 231-239, 2005.

[33] J. A. Detre, L. Maccotta, D. King et al., "Functional MRI lateralization of memory in temporal lobe epilepsy," Neurology, vol. 50, no. 4, pp. 926-932, 1998.

[34] M. L. Rabin, V. M. Narayan, D. Y. Kimberg et al., "Functional MRI predicts post-surgical memory following temporal lobectomy," Brain, vol. 127, no. 10, pp. 2286-2298, 2004.
[35] M. P. Richardson, B. A. Strange, J. S. Duncan, and R. J. Dolan, "Memory fMRI in left hippocampal sclerosis: optimizing the approach to predicting postsurgical memory," Neurology, vol. 66, no. 5, pp. 699-705, 2006.

[36] J. R. Binder, S. J. Swanson, D. S. Sabsevitz, T. A. Hammeke, M. Raghavan, and W. M. Mueller, "A comparison of two fMRI methods for predicting verbal memory decline after left temporal lobectomy: language lateralization versus hippocampal activation asymmetry," Epilepsia, vol. 51, no. 4, pp. 618-626, 2010.

[37] M. P. Richardson, B. A. Strange, and R. J. Dolan, "Encoding of emotional memories depends on amygdala and hippocampus and their interactions," Nature Neuroscience, vol. 7, no. 3, pp. 278-285, 2004.

[38] M. P. Richardson, B. A. Strange, P. J. Thompson, S. A. Baxendale, J. S. Duncan, and R. J. Dolan, "Pre-operative verbal memory fMRI predicts post-operative memory decline after left temporal lobe resection," Brain, vol. 127, no. 11, pp. 24192426, 2004.

[39] S. B. Bonelli, R. H. W. Powell, M. Yogarajah et al., "Imaging memory in temporal lobe epilepsy: predicting the effects of temporal lobe resection," Brain, vol. 133, no. 4, pp. 1186-1199, 2010.

[40] J. R. Binder, D. S. Sabsevitz, S. J. Swanson, T. A. Hammeke, M. Raghavan, and W. M. Mueller, "Use of preoperative functional MRI to predict verbal memory decline after temporal lobe epilepsy surgery," Epilepsia, vol. 49, no. 8, pp. 1377-1394, 2008.

[41] K. Deblaere, W. H. Backes, P. Hofman et al., "Developing a comprehensive presurgical functional MRI protocol for patients with intractable temporal lobe epilepsy: a pilot study," Neuroradiology, vol. 44, no. 8, pp. 667-673, 2002.

[42] R. T. Constable, A. Carpentier, K. Pugh, M. Westerveld, Y. Oszunar, and D. D. Spencer, "Investigation of the human hippocampal formation using a randomized event-related paradigm and Z-shimmed functional MRI," NeuroImage, vol. 12, no. 1, pp. 55-62, 2000.

[43] D. R. Addis, M. Moscovitch, and M. P. McAndrews, "Consequences of hippocampal damage across the autobiographical memory network in left temporal lobe epilepsy," Brain, vol. 130, no. 9, pp. 2327-2342, 2007.

[44] K. Henke, A. Buck, B. Weber, and H. G. Wieser, "Human hippocampus establishes associations in memory," Hippocampus, vol. 7, no. 3, pp. 249-256, 1997.

[45] N. M. Hunkin, A. R. Mayes, L. J. Gregory et al., "Noveltyrelated activation within the medial temporal lobes," Neuropsychologia, vol. 40, no. 8, pp. 1456-1464, 2002.

[46] T. Grunwald, K. Lehnertz, H. J. Heinze, C. Helmstaedter, and C. E. Elger, "Verbal novelty detection within the human hippocampus proper," Proceedings of the National Academy of Sciences of the United States of America, vol. 95, no. 6, pp. 31933197, 1998.

[47] E. Tulving, H. J. Markowitsch, F. E. Craik, R. Habib, and S. Houle, "Novelty and familiarity activations in PET studies of memory encoding and retrieval," Cerebral Cortex, vol. 6, no. 1, pp. 71-79, 1996.

[48] J. R. Binder, P. S. F. Bellgowan, T. A. Hammeke, E. T. Possing, and J. A. Frost, "A comparison of two fMRI protocols for eliciting hippocampal activation," Epilepsia, vol. 46, no. 7, pp. 1061-1070, 2005.

[49] H. W. R. Powell, M. P. Richardson, M. R. Symms et al., "Preoperative fMRI predicts memory decline following anterior temporal lobe resection," Journal of Neurology, Neurosurgery and Psychiatry, vol. 79, no. 6, pp. 686-693, 2008. 
[50] L. Frings, K. Wagner, U. Halsband, R. Schwarzwald, J. Zentner, and A. Schulze-Bonhage, "Lateralization of hippocampal activation differs between left and right temporal lobe epilepsy patients and correlates with postsurgical verbal learning decrement," Epilepsy Research, vol. 78, no. 2-3, pp. 161-170, 2008.

[51] S. Dupont, E. Duron, S. Samson et al., "Functional MR imaging or wada test: which is the better predictor of individual postoperative memory outcome?" Radiology, vol. 255, no. 1, pp. 128-134, 2010.

[52] H. W. R. Powell, M. P. Richardson, M. R. Symms et al., "Reorganization of verbal and nonverbal memory in temporal lobe epilepsy due to unilateral hippocampal sclerosis," Epilepsia, vol. 48, no. 8, pp. 1512-1525, 2007.

[53] T. Loddenkemper, H. H. Morris, and G. Möddel, "Complications during the Wada test," Epilepsy and Behavior, vol. 13, no. 3, pp. 551-553, 2008.

[54] J. A. Wada, "A new method for the lateralization of the side of cerebral speech dominance: a preliminary report on the intracranial injection of sodium amytal in man," Igaku Seibutsug$a k u$, vol. 14, pp. 221-222, 1949.

[55] J. A. Wada, "Clinical experimental observations of carotid artery injections of sodium amytal," Brain and Cognition, vol. 33, no. 1, pp. 11-13, 1997.

[56] B. Milner, C. Branch, and T. Rassmussen, "Study of shortterm memory after intracarotid injection of sodium amytal," Transactions of the American Neurological Association, vol. 87, pp. 224-226, 1962.

[57] G. J. Chelune, R. I. Naugle, H. Luders, and I. A. Awad, "Prediction of cognitive change as a function of preoperative ability status among temporal lobectomy patients seen at 6-month follow-up," Neurology, vol. 41, no. 3, pp. 399-404, 1991.

[58] T. T. Lineweaver, H. H. Morris, R. I. Naugle, I. M. Najm, B. Diehl, and W. Bingaman, "Evaluating the contributions of state-of-the-art assessment techniques to predicting memory outcome after unilateral anterior temporal lobectomy," Epilepsia, vol. 47, no. 11, pp. 1895-1903, 2006.

[59] N. Elshorst, B. Pohlmann-Eden, S. Horstmann, R. Schulz, F. Woermann, and M. P. McAndrews, "Postoperative memory prediction in left temporal lobe epilepsy: the Wada test is of no added value to preoperative neuropsychological assessment and MRI," Epilepsy and Behavior, vol. 16, no. 2, pp. 335-340, 2009.

[60] B. P. Hermann, M. Seidenberg, A. Haltiner, and A. R. Wyler, "Relationship of age at onset, chronologic age, and adequacy of preoperative performance to verbal memory change after anterior temporal lobectomy," Epilepsia, vol. 36, no. 2, pp. 137-145, 1995.

[61] A. J. Saykin, R. C. Gur, N. M. Sussman, M. J. O’Connor, and R. E. Gur, "Memory deficits before and after temporal lobectomy: effect of laterality and age of onset," Brain and Cognition, vol. 9, no. 2, pp. 191-200, 1989.

[62] M. R. Trenerry, C. R. Jack, R. J. Ivnik et al., "MRI hippocampal volumes and memory function before and after temporal lobectomy," Neurology, vol. 43, no. 9, pp. 1800-1805, 1993.

[63] A. A. Cohen-Gadol, M. Westerveld, J. Alvarez-Carilles, and D. D. Spencer, "Intracarotid Amytal memory test and hippocampal magnetic resonance imaging volumetry: validity of the Wada test as an indicator of hippocampal integrity among candidates for epilepsy surgery," Journal of Neurosurgery, vol. 101, no. 6, pp. 926-931, 2004.

[64] J. R. White, D. Matchinsky, T. E. Beniak et al., "Predictors of postoperative memory function after left anterior temporal lobectomy," Epilepsy and Behavior, vol. 3, no. 4, pp. 383-389, 2002.

[65] A. C. Kneebone, G. J. Chelune, D. S. Dinner, R. I. Naugle, and I. A. Awad, "Intracarotid amobarbital procedure as a predictor of material-specific memory change after anterior temporal lobectomy," Epilepsia, vol. 36, no. 9, pp. 857-865, 1995.

[66] F. Andelman, S. Kipervasser, M. Y. Neufeld, U. Kramer, and I. Fried, "Predictive value of Wada memory scores on postoperative learning and memory abilities in patients with intractable epilepsy," Journal of Neurosurgery, vol. 104, no. 1, pp. 20-26, 2006.

[67] K. J. Meador and D. W. Loring, "The Wada test: controversies, concerns, and insights," Neurology, vol. 52, no. 8, pp. 15351536, 1999.

[68] B. Abou-Khalil and B. L. Schlaggar, "Is it time to replace the Wada test?” Neurology, vol. 59, no. 2, pp. 160-161, 2002.

[69] H. E. Kirsch, J. A. Walker, F. S. Winstanley et al., "Limitations of Wada memory asymmetry as a predictor of outcomes after temporal lobectomy," Neurology, vol. 65, no. 5, pp. 676-680, 2005.

[70] C. L. Grote and K. Meador, "Has amobarbital expired? Considering the future of the Wada," Neurology, vol. 65, no. 11, pp. 1692-1693, 2005. 


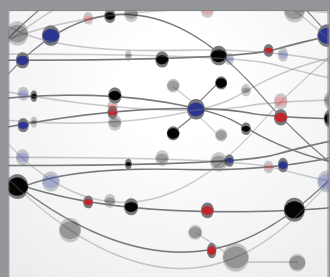

The Scientific World Journal
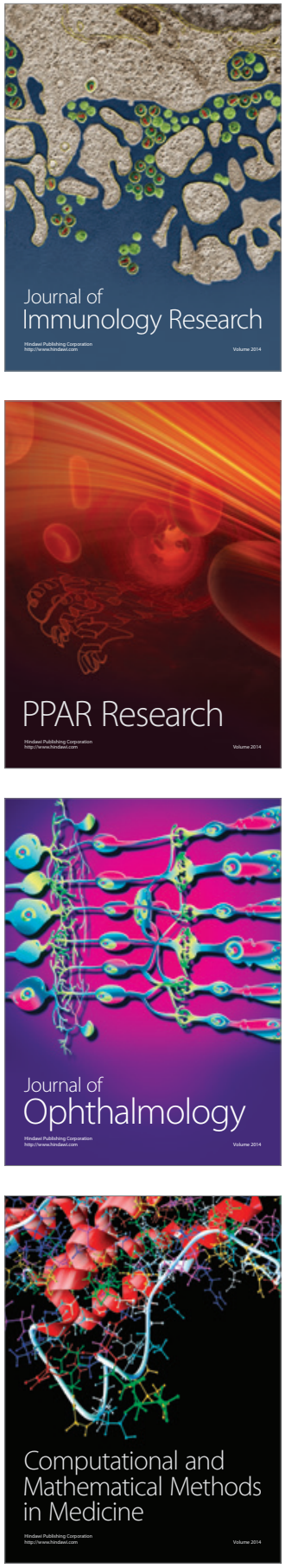

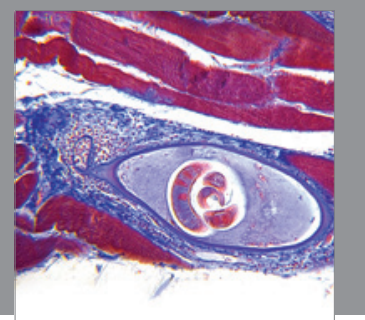

Gastroenterology

Research and Practice
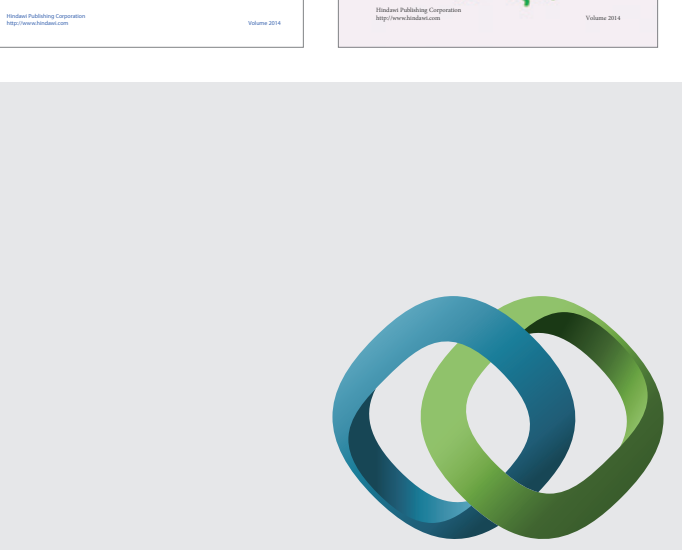

\section{Hindawi}

Submit your manuscripts at

http://www.hindawi.com
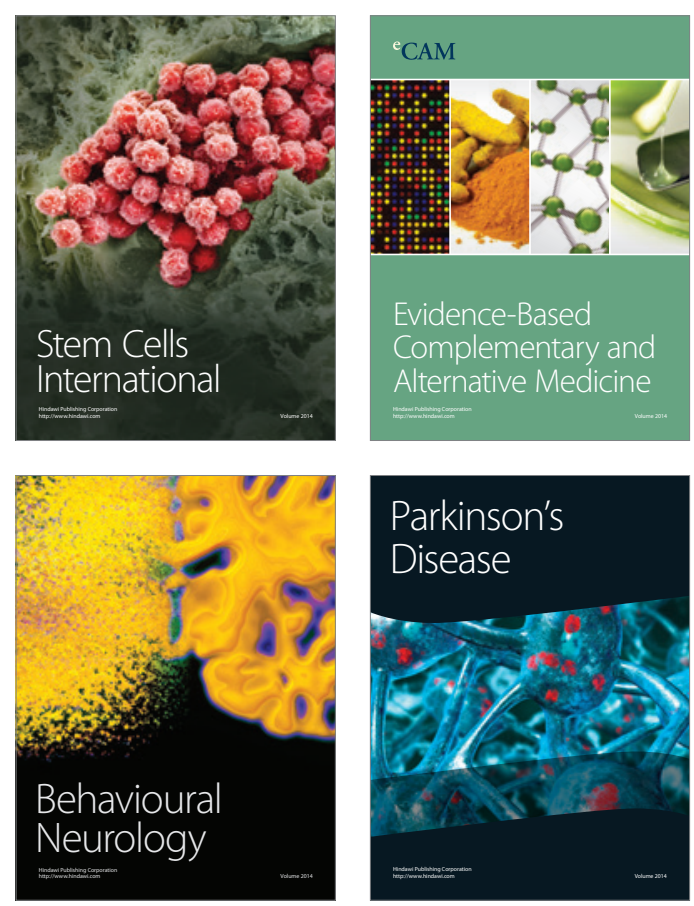

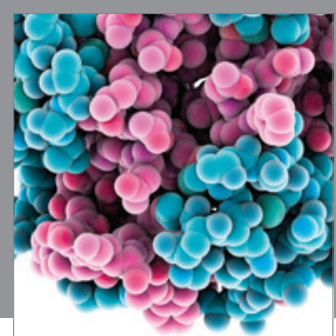

Journal of
Diabetes Research

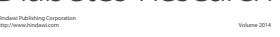

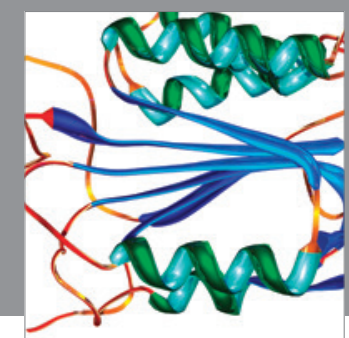

Disease Markers
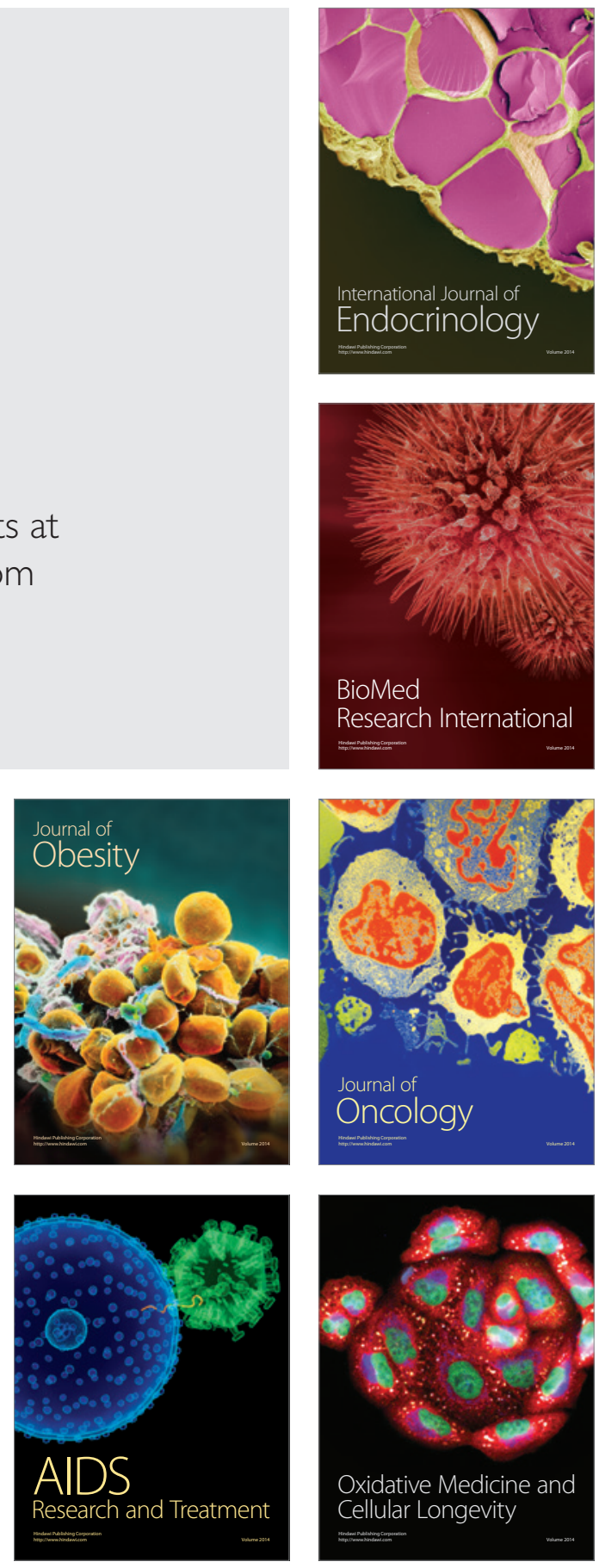